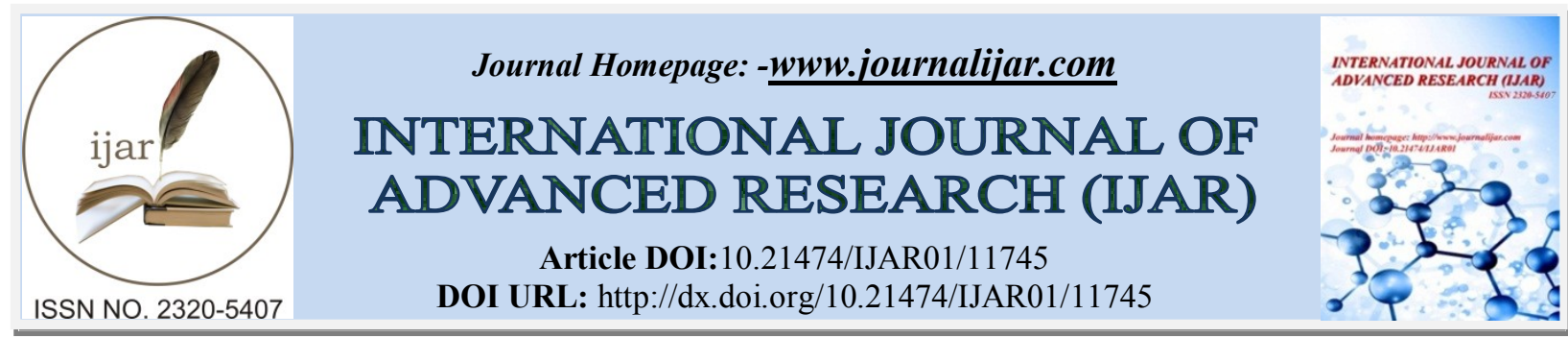

RESEARCH ARTICLE

\title{
UNE LOCALISATION RARE D'UN SCHWANNOME ORBITAIRE A RARE LOCALIZATION OF AN ORBITAL SCHWANNOMA
}

Zahra Sayad, Bouchra Dani and Malik Boulaadas

\section{Manuscript Info}

\section{Manuscript History}

Received: 20 July 2020

Final Accepted: 24 August 2020

Published: September 2020

Key words:-

Schwannoma, Infraorbital Nerve, Orbital Floor

\begin{abstract}
Introduction: The infraorbital schwannoma or neuroma is a rare benign tumor of the orbit. Its clinical and radiological presentation is not very specific. Its diagnosis is essentially histological.

Observation: We report the case of a 46-year-old woman, diabetic under treatment, consulting for an irreducible, non-axial and nonpulsatile exophthalmos. Clinical examination of the left eye found visual acuity corrected to $8 / 10$ without limitation of ocular motility or diplopia. The examination of the right eye is unremarkable. The orbital CT objectified a mass measuring $25.3 \mathrm{~mm} \times 13.8 \mathrm{~mm}$, homogeneous of the floor of the left orbit, pushing the eyeball up and out. The orbital floor was pushed inferiorly by the mass; however, its integrity was preserved. Tumor excision was performed via the sub-ciliary cutaneous incision with reconstruction of the floor by an iliac crest graft. The histological analysis allowed us to have a definite diagnosis of schwannoma.

Conclusion:The schwannoma is a rare tumor of the orbit. Its diagnosis is established solely by anatomopathological study of the operative specimen. Its treatment is based on a complete surgical excision to avoid any recurrence.
\end{abstract}

Copy Right, IJAR, 2020, All rights reserved.

\section{Introduction:-}

Le schwannome ou neurilemome orbitaire est une tumeur bénigne rare de l'orbite ( 1 à $4 \%$ des tumeurs orbitaires). Son diagnostic repose sur l'étude histologique de la lésion. Nous rapportons à travers cetteobservation les particularités cliniques, d'imagerie et chirurgicales d'une localisation rare de cette tumeur. $[1,2]$

\section{Observation:-}

Une patiente âgée de 46 ans, ayant comme antécédent un diabète type 2 sous antidiabétique oral, admise pour une exophtalmie gauche non axiale déviée en haut et légèrement en dehors, non pulsatile, irréductible et indolore évoluant depuis deux ans.

L'examen de l'œil gauche retrouve une acuité visuelle corrigée à $8 / 10$, pas de masse palpable avec une motilité oculomotrice conservée. L'examen de l'œil droit est sans particularités. Le reste de l'somatique examen était normal.

\section{Corresponding Author:- Zahra Sayad}


Une tomodensitométrie orbitaire (Fig. 1) a montré un processus lésionnel tissulaire isodense au parenchyme cérébral, mesurant $25,3 \mathrm{~mm} \times 13,8 \mathrm{~mm}$, homogène, non rehaussé après injection du produit de contraste, encapsulée et bien limité n'envahissant pas les structures adjacentes qui se développe au niveau du plancher de l'orbite gauche.

Cette formation refoule le globe oculaire sans l'envahir en haut et légèrement en dehors responsable d'une exophtalmie grade II et soufflant le plancher en bas.

La patiente a bénéficiée d'une résection totale de la tumeur par voie d'abord sous-ciliaire, avec reconstruction du plancher de l'orbite par un greffon de crête iliaque. (Fig. 2 et 3)

L'examen anatomopathologique de la pièce opératoire obtenue a permis de poser le diagnostic d'un schwannome orbitaire développé probablement aux dépens du nerf sous orbitaire.

Les suites opératoires étaient simples mises à part l'hypoesthésie au territoire infra orbitaire. Sur un recul de 24 mois, aucune récidive n'a été décelée.

\section{Discussion:-}

Le schwannome orbitaire est une tumeur nerveuse bénigne rare qui représente 1-5\% des tumeurs orbitaires de la gaine de Schwann des nerfs périphériques.Moins de $1 \%$ subissent une transformation maligne en neurofibrosarcome. $[2,3]$

C'est une pathologie de l'adulte entre 20 et 65 ans, avec une prédominance féminine, le cas de notre patiente. [1,2,3]

Cette tumeur est caractérisée par une croissance lente. Sa présentation clinique dépend de la localisation de la tumeur et de la compression des structures adjacentes, dominée par une exophtalmie lentement progressive et retentissant peu sur la vision et la motilité oculaire. Les signes inflammatoires et les douleurs sont rares. [3,4]

Le schwannome provenant du nerf sous-orbitaire est très rare. Ce sont les nerfs supra-orbitaire et supra-trochléaire qui sont les plus touchés. Cependant dans $50 \%$ des cas le nerf d'origine reste inconnu. Dans notre cas, c'est l'hypoesthésie infra orbitaire postopératoire qui nous a confirmé le nerf à l'origine de cette tumeur. [5,6]

L'imagerie permet de déterminer le type de lésion, sa localisation et ses rapports avec les structures adjacentes. La tomodensitométrie retrouve une masse solitaire du plancher de l'orbite, tissulaire, bien limitée, iso dense avec le cerveau, encapsulée et habituellement hétérogène, de rehaussement variable. $[3,7,8]$

L'imagerie par résonance magnétique montre un isosignal $\mathrm{T} 1$ et un hypersignal $\mathrm{T} 2$ avec un rehaussement variable après injection de gadolinium. [3]

Le diagnostic est confirmé par l'histologie qui montre une tumeur encapsulée, souvent hétérogène avec fréquemment des zones kystiques. L'immuno-histochimie permet d'individualiser le schwannome des autres tumeurs à cellules fusiformes. On note une forte expression de l'antigène PS 100 : marqueur tumoral sécrété par les cellules nerveuses. [7,9]

Le traitement est exclusivement chirurgical, et consiste en une exérèse totale en monobloc pour éviter tout risque de récidive ou de dégénérescence maligne malgré qu'elle soit exceptionnelle justifiant ainsi un suivi régulier. [7,10]

La voie d'abord chirurgicale est déterminée par la topographie de la lésion. Dans notre cas, c'était une voie d'abord sous ciliaire, qui nous a permis une exérèse complète de la tumeur et la reconstruction de la dépression résiduelle au niveau du plancher orbitaire par un greffon autologue de la crête iliaque. Son pronostic est bon après une exérèse chirurgicale complète. [7]

\section{Conclusion:-}

Le schwannomeinfraorbitaire reste une entité très rare. La clinique est peu contributive tandis que seule l'étude histologique et immuno-histichimique qui permet d'établir le diagnostic. Sa prise en charge repose sur l'exérèse chirurgicale complète avec un suivi clinique prolongé. 


\section{Conflits d'intérêts :}

Les auteurs ne déclarent aucun conflit d'intérêts.

\section{Figures :}

1. R. El Halimi*, H. Kharbouch, M. CherifChefchaouni, A. El Hassan, F. Bencherifa, A. Berraho. Schwannome orbitaire : aspects cliniques, radiologiques et chirurgicaux. J Fr Ophtal (2013) 36, 160-163.

2. Abdelhay Zouak1,2, Maryame Abou-Elfad11, Mohamed Roubal1, Mohamed Mahtar. Un cas de schwannome orbitaire. Les Cahiers d'Ophtalmologie 2014; $n^{\circ} 185: 28-9$

3. Ech-Cherif El Kettani N, El Quessar A, El Hassani MR et al. Une cause rare d'exophtalmie: le schwannome bénin orbitaire. J Radiol. 2007; 88:282-4

4. Kiratli H, Erkan K, Söylemezologlu F. Schwannomes orbitaires isolés : aspects cliniques, radiologiques et chirurgicaux. J Fr Ophtal - mol. 2007 ;30:986-91.

5. Mesut Sabri Tezer a, *, MugeOzcan b , O* zge Han a , Adnan Unal a , SametOzlugedik. Schwannoma originating from the infraorbital nerve: A case report. Auris Nasus Larynx 33 (2006) 343-345

6. Ha W, Lee JW, Choi J, Yang SW, Kim SY. Schwannoma originating from infraorbital nerve. Arch Craniofac Surg 2013; 14:61-4.

7. Stephanie M. Young, FAMS, FRCOphth, Yoon-Duck Kim, MD, PhD,y Sung Soon Hwang, MD,y and Kyung In Woo, MD, PhDy. Orbital Schwannoma with Atypical Presentation. The Journal of Craniofacial Surgery Vol 00, N 00, Month 2018

8. Kumar Nilesh. Infra-orbital nerve schwannoma: Report and review. Journal of Natural Science, Biology and Medicine | January 2015 | Vol 6 | Issue 1

9. Colapinto P, Sheth HG, Jain R, et al. Inferior oblique schwannoma: diagnosis and management. Orbit 2007; 26:287-289

10. Ma KK, Callahan AB, Wang SJ, et al. Atypical rapidly enlarging orbital schwannoma. OphthalPlastReconstr Surg 2017;33: S111-S114

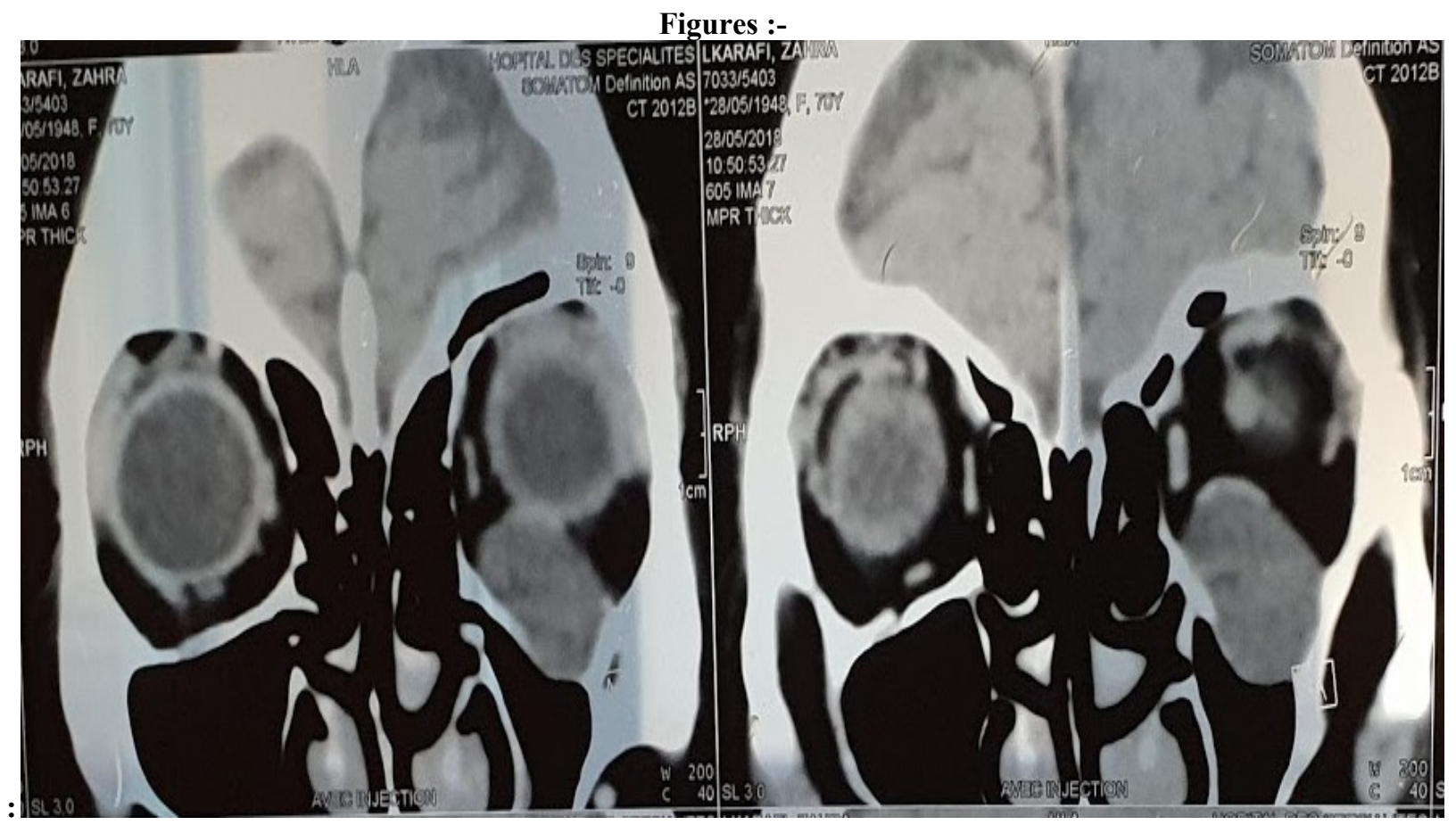

Figure 1:- TDM orbitaire en coupe coronale, fenêtre parenchymateuse montrant un processus tissulaire du plancher de l'orbite gauche, bien limité, refoulant le globe oculaire en haut et en dehors et soufflant le plancher en bas. 


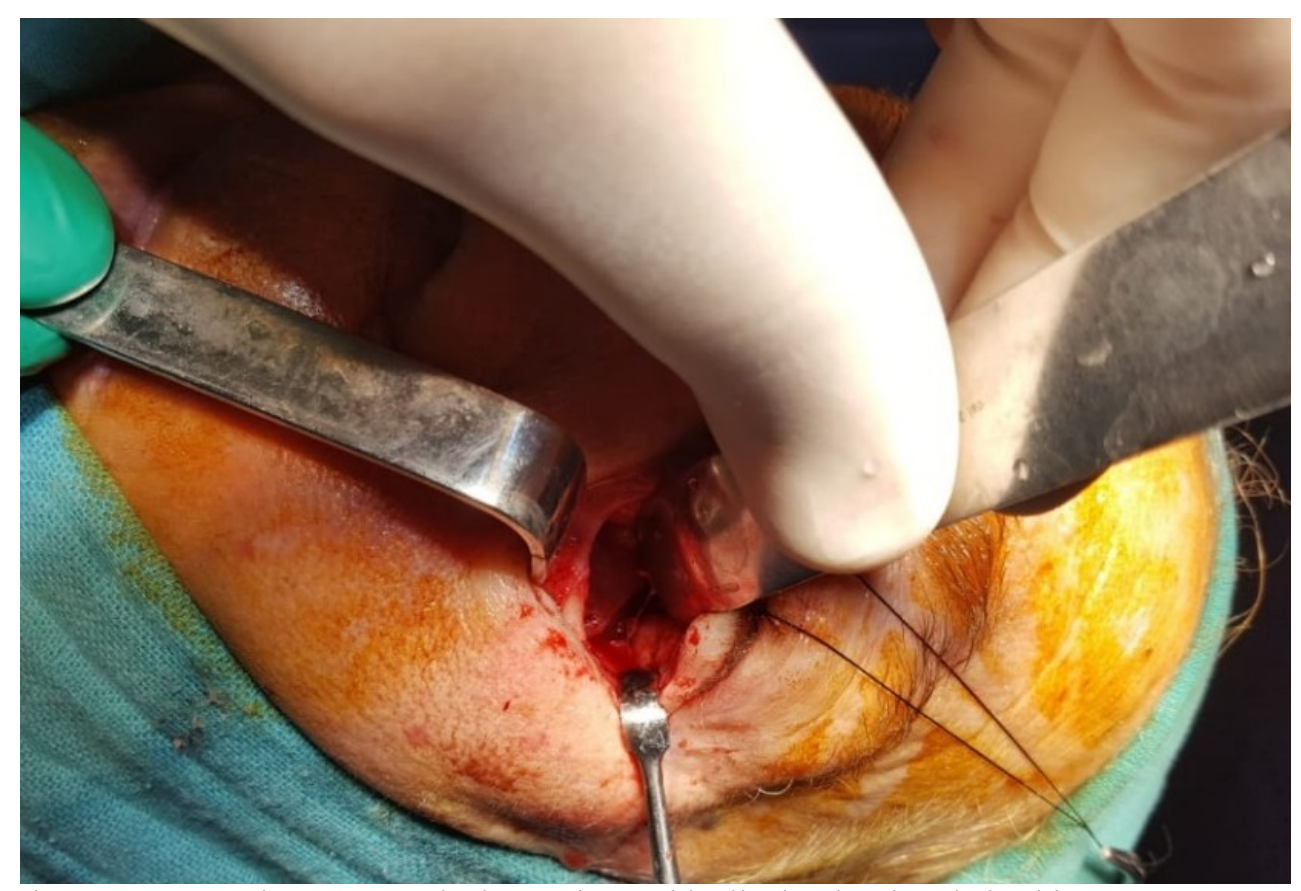

Figure 2:- photo per opératoire montrant la dépression résiduelle du plancher de l'orbite après exérèse de la masse par voie d'abord sous ciliaire.

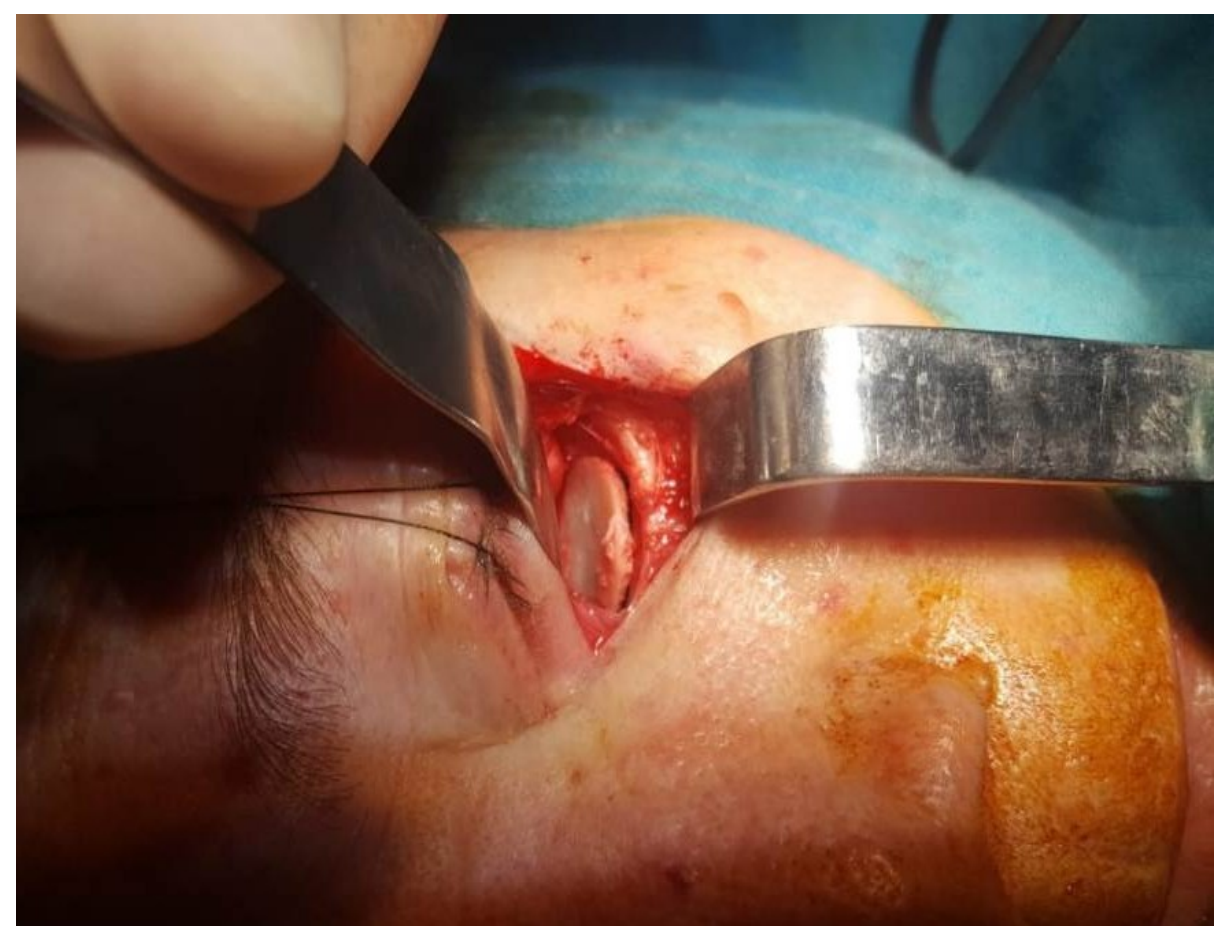

Figure 3:- photo per opératoire montrant la reconstruction du plancher de l'orbite par un greffon de la crête iliaque. 